\title{
Urothelial Carcinoma of the Bladder and the Rise of Immunotherapy
}

\author{
David D. Chism, MD, MS
}

\begin{abstract}
With the advent of platinum-based chemotherapy, survival rates for metastatic urothelial carcinoma have plateaued, giving way to the modern immunotherapy paradigm. Although immunotherapy as an effective treatment dates back to the live, attenuated bacillus Calmette-Guérin vaccine, the recent impact of immune checkpoint inhibitors targeting programmed death/programmed death-ligand 1 (PD-1/PD-L1) coupled with the promise of both anti-cytotoxic T-lymphocyte-associated protein 4 (CTLA-4) antibodies and indoleamine-2, 3-dioxygenase-1 (IDO-1) inhibitors have provided a resurgence. To date, pembrolizumab, a PD-1 inhibitor, has been granted full FDA approval based on its high antitumor activity, tolerability, and efficacy, with notable prolonged durable responses in the second-line setting. Nivolumab (a PD-1 inhibitor) and atezolizumab, durvalumab, and avelumab (PD-L1 inhibitors) have also gained accelerated drug approval in the second-line setting. In addition, atezolizumab and pembrolizumab have been approved for platinum-ineligible patients in the firstline setting. Effective 2-drug combinations reported include nivolumab plus the CTLA-4 antibody ipilimumab and pembrolizumab plus the IDO-1 inhibitor epacadostat. Further expansion of immunotherapy will hinge in part on the ability to define responders versus nonresponders through the use of biomarkers like PD-L1 or mutational load. Clinical trials with immunotherapy for metastatic disease as single agents or in combination are ongoing. This review explores the rise of immunotherapy and presents the current treatments and challenges posed with development of biomarkers, and provides a summary of ongoing phase III clinical trials.
\end{abstract}

J Natl Compr Canc Netw 2017;15(10):1277-1284 doi: 10.6004/jncen.2017.7036

$\mathrm{U}_{\text {rothelial carcinoma }}(\mathrm{UC})$ of the bladder is projected to account for 79,030 cases and 16,870 deaths in 2017 in the United States. ${ }^{1}$ Median age at diagnosis is 73 years, with main risk factors including smoking, age, and male sex. Of the $70 \%$ of patients diagnosed with non-muscle-invasive bladder cancer (N-MIBC), nearly a quarter experience progression to muscle-invasive bladder cancer (MIBC), which is the more aggressive phenotype. Approximately one-third of newly diagnosed patients will have MIBC at time of diagnosis, and approximately $10 \%$ will have regional lymph node-positive or distant metastatic disease at presentation, with 5-year survival rates of $35 \%$ and $5 \%$, respectively. ${ }^{2}$

Cisplatin-based combination chemotherapy remains the cornerstone of treatment for medically fit pa-

From the Division of Hematology and Oncology, Department of Medicine, Vanderbilt-Ingram Cancer Center, Vanderbilt University Medical Center, Nashville, Tennessee.

Submitted July 17, 2017; accepted for publication September 11, 2017. tients with metastatic disease as defined by the consensus group working definition. ${ }^{3}$ Standard methotrexate, vinblastine, doxorubicin, and cisplatin (MVAC) every 4 weeks compared with cisplatin alone resulted in a median overall survival (OS) of 12.5 versus 8.2 months, albeit with greater toxicity. ${ }^{4} \mathrm{~A}$ phase III randomized trial comparing dose-dense MVAC with granulocyte colonystimulating factor (G-CSF) every 2 weeks versus standard MVAC showed a benefit in progression-free survival (PFS; 9.1 vs 8.2 months; $P=.037$ ) with fewer dose delays and less toxicity., ${ }^{5,6}$ Therefore, standard MVAC is no longer recommended for first-line treatment for bladder cancer. The gemcitabine plus cisplatin (GC) doublet and the paclitaxel, cisplatin, plus gemcitabine (PCG) triplet are also effective treatments based on median survivals of 14 and 16 months, respectively. ${ }^{7,8}$ Dose-

Dr. Chism has disclosed that he serves on a scientific advisory board for Exelixis, Inc. and Karyopharm Therapeutics Inc.

Correspondence: David D. Chism, MD, MS, Vanderbilt University Medical Center, 2220 Pierce Avenue, 792 Preston Research Building, Nashville, TN 37232. E-mail: david.d.chism@vanderbilt.edu 
dense MVAC plus G-CSF, the GC doublet, and the PCG triplet are NCCN Clinical Practice Guidelines in Oncology (NCCN Guidelines) category 1 recommendations for first-line treatment of metastatic disease. ${ }^{9}$ For those unfit for cisplatin, carboplatin-based alternative combinations result in a median OS of 9 months. ${ }^{10}$ Vinflunine was approved in Europe for second-line treatment based on a phase III trial demonstrating a 2-month survival benefit over best supportive care (BSC). ${ }^{11}$ Salvage single agents produce a modest overall response rate (ORR) between 10\% to $20 \%$, median PFS of 2 to 3 months, and median OS of 6 to 9 months. ${ }^{12-14}$

Both the unmet need for second-line therapies for bladder cancer and a resurgence of immunotherapy in other solid tumors coincided to provide the backdrop for the immunotherapy launch into bladder cancer. Ipilimumab, a monoclonal antibody to cytotoxic T-lymphocyte antigen 4 (CTLA-4) and a negative regulator of $\mathrm{T}$-cell activation, became the first agent to demonstrate an OS advantage and received FDA approval in 2011 for first-line treatment of melanoma. ${ }^{15}$ Furthermore, the results of a large phase III trial demonstrated that ipilimumab plus nivolumab and nivolumab alone resulted in a significant PFS benefit over ipilimumab alone. ${ }^{16}$ Pembrolizumab is also an option based on its improved response and PFS compared with chemotherapy or ipilimumab, and its lower rate of adverse events (AEs) and improved OS when compared with ipilimumab alone. ${ }^{17}$ Both nivolumab or pembrolizumab alone are category 1 recommendations for metastatic melanoma; ipilimumab plus nivolumab can also be considered with careful patient selection. Nivolumab was also FDA-approved for advanced squamous non-small cell lung cancer (NSCLC) and nonsquamous cell NSCLC in the second-line setting, regardless of PD-L1 protein expression. Pembrolizum$\mathrm{ab}$ was approved in the first-line setting for patients with NSCLC with high PD-L1 expression and without expression of EGFR or ALK aberrations. Like melanoma and lung cancer, bladder cancer also has a high mutational load that may confer increased immunogenicity, providing strong rationale for its further development. ${ }^{18}$

\section{Rationale for Immunotherapy}

The PD-1/PD-L1/2 axis emerged as a therapeutic avenue due to its critical role in the escape from immune surveillance. Under normal conditions, PD-1 is usually expressed on the surface of activated $\mathrm{T}$ cells and functions as a pathway to modulate excessive immune reactions. Its ligands, PD-L1 and PDL2, are found on a variety of normal tissues, with the PD-1 and PD-L2 interaction having a critical role in maintaining peripheral tolerance. In UC, the upregulation of PD-1 and PD-L1 promotes peripheral T-cell exhaustion (termed adaptive immune resistance). ${ }^{19}$ PD-1 and B7.1 (CD80) serve as negative regulators of T-lymphocyte activation. Monoclonal antibodies to PD-1 inhibit binding to the PD-1 receptor to block the ligands PD-L1 and PD-L2 from binding; PD-L1 monoclonal antibodies block PD-L1 expressed on tumor cells and tumor-infiltrating cells, disrupting the interaction of PD-1 and B7.1 or the CD80 receptor. Blocking the negative PD-1 receptor signaling restores antitumor T-cell function by promoting T-cell migration, proliferation, and secretion of cytotoxic mediators.

Like PD-1, CTLA-4 is an inhibitory receptor expressed on $\mathrm{T}$ cells with a higher binding affinity to the costimulatory receptor B7.1 than to the costimulatory receptor CD28. The stronger binding between CTLA-4 to B7.1 than CD28 and B7 favors inhibitory signaling, resulting in tumor evasion. Ipilimumab and tremelimumab (also a CTLA-4 inhibitor) block this interaction restoring antitumor cell function. Indoleamine-2,3-dioxygenase 1 (IDO1) is an intracellular immunoregulatory rate-limiting enzyme that contributes to immunosuppression, tolerance, and tumor escape by catabolizing tryptophan to kynurenines. Tryptophan depletion and production of kynurenines and other metabolites shift the immune microenvironment to an immunosuppressive state. ${ }^{20}$ Blocking IDO1 results in increased tryptophan, thus yielding increased antitumor activity (Figure 1).

\section{Atezolizumab (MPDL3280A)}

Atezolizumab, an engineered humanized immunoglobulin G1 monoclonal antibody immune checkpoint inhibitor to PD-L1, was the first immunotherapy checkpoint inhibitor approved by the FDA based on the results of IMvigor 210, a multicenter, single-arm, 2-cohort, phase II trial in patients with 


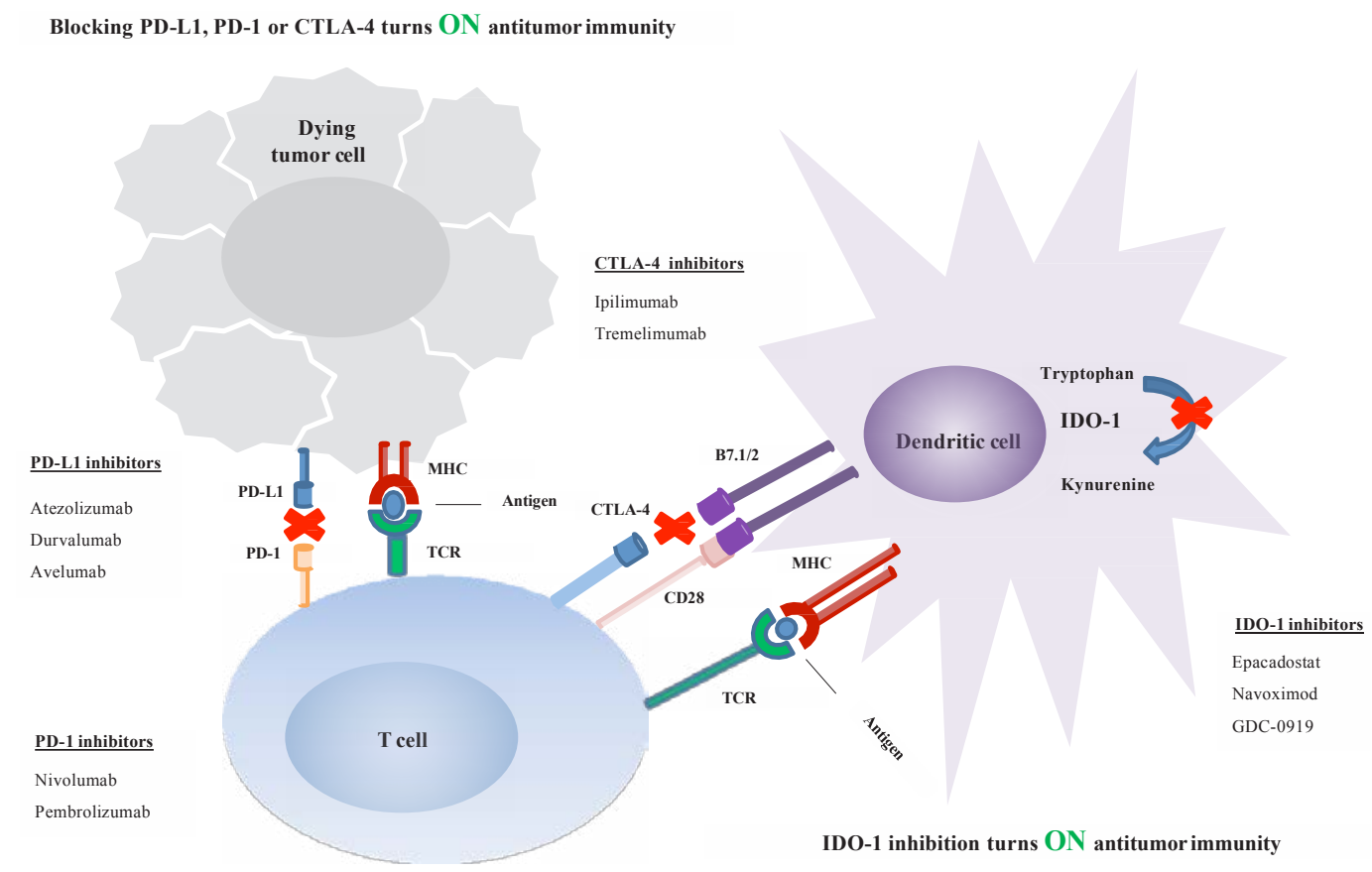

Figure 1. Key immunotherapy mechanisms of urothelial carcinoma of the bladder.

Abbreviations: CTLA-4, cytotoxic T-lymphocyte-associated protein 4; IDO, indoleamine-2, 3-dioxygenase-1; PD-1, programmed death-1; PD-L1, programmed death-ligand 1; TCR, T-cell receptor.

metastatic UC that progressed after platinum-based therapy. The second cohort of the phase II study examined atezolizumab (1,200 mg intervenously every 3 weeks) in patients whose disease progressed after platinum-based treatment, stratified by PD-L1 expression measured on infiltrating immune cells. The ORR was compared with a historical control response rate of $10 \%$ in the second-line setting. Results showed a significantly improved ORR of $26 \%$ (95\% CI, 18-36) in 100 of 310 patients with high PD-L1 expression and a 15\% ORR (5\% complete response [CR]) in all patients with a median followup of 11.7 months (95\% CI, 11.4-12.2), with durable responses. ${ }^{21}$ Common treatment-related AEs (TRAEs) included fatigue, pneumonitis, rash, and dyspnea. Among the 119 cisplatin-ineligible patients in cohort 1 who received atezolizumab in the first-line setting, there was an ORR of 23\% (95\% CI, 16-31) and a CR of 9\%, with durable responses. Responses occurred across all PD-L1 stratifications with the median OS at 15.9 months. These results compare favorably with those of carboplatin-based alternatives for patients ineligible for cisplatin, with a median OS of 9 months. TRAEs were minimal and included fatigue, pruritus, and diarrhea. ${ }^{22}$
IMvigor 211 was a phase III randomized study that compared atezolizumab versus investigator's choice of chemotherapy (vinflunine vs taxanes) in 931 patients in the second-line setting. Crossover was not permitted. OS of patients with high PDL1 expression, the primary end point of the study, was not significantly different for atezolizumab versus chemotherapy (11.1 vs 10.6 months; hazard ratio [HR], 0.87; $P=.41)$. However, an improved OS in patients treated with atezolizumab versus chemotherapy, regardless of PD-L1 expression, was observed ( 8.6 vs 8.0 months; HR, $0.85 ; P=.038$ ) with a median follow-up of 17.3 months. Atezolizumab also demonstrated a more favorable safety profile than chemotherapy. ${ }^{23}$ Atezolizumab is supported by the NCCN Guidelines for treatment in both the firstline and second-line settings for patients ineligible for cisplatin-based chemotherapy. ${ }^{9}$

\section{Durvalumab (MED14736)}

Durvalumab, a human immunoglobin G1к monoclonal antibody that binds PD-L1, gained FDA accelerated approval in May 2017 and is also recommended by the NCCN Guidelines for treatment in the second-line setting. ${ }^{9}$ In a phase I/II trial, 61 patients received durvalumab $(10 \mathrm{mg} / \mathrm{kg}$ every 2 weeks 
intravenously), with the initial 20 patients enrolled regardless of PD-1 expression; however, subsequent patients were required to have a minimum of $5 \%$ PD-1 expression on tumor cells. There was a $31 \%$ ORR (95\% CI, 17.6-47.1), with responses ongoing in 12 of 13 responding patients with minimal treatment-related side effects. The most common TRAEs included fatigue, diarrhea, and decreased appetite. When examining response based on PD-L1 expression, the ORR was $46.4 \%$ in the PD-L1-positive group and $0 \%$ in the PD-L1-negative group. ${ }^{24} \mathrm{PD}$ L1 expression in this study, which was measured on both immune and tumor cells, was associated with improved response to durvalumab. A planned update on the safety and efficacy was reported on 191 patients with a median follow-up of 5.78 months; the ORR was $17.8 \%$ including 7 CRs, with responses observed regardless of PD-L1 status, and the median OS was 18.2 months. ${ }^{25}$

\section{Avelumab}

Avelumab, a human IgG1 monoclonal antibody for PD-L1 administered at $10 \mathrm{mg} / \mathrm{kg}$ intravenously every 2 weeks, gained FDA accelerated approval in May 2017 and is recommended by the NCCN Guidelines as second-line treatment for metastatic UC. A phase Ib expansion cohort of 44 patients with metastatic UC treated with avelumab in the second-line setting was performed with safety and tolerability as its primary end points. Similar to both durvalumab, avelumab was well tolerated, with most frequent TRAEs being fatigue, infusion-related reactions, and nausea. With a median follow-up of 16.5 months, the confirmed central reviewed ORR was $18.2 \%$ (5 CRs), with a median OS of 13.7 months. ${ }^{26}$ The exploratory marker PD-L1 also trended toward greater clinical activity, with 7 of the 8 patients with PD-L1-positive tumors experiencing a response to avelumab.

\section{Nivolumab}

Nivolumab, a fully human monoclonal IgG4 antibody that blocks PD-1, was FDA-approved for use in the second-line setting for metastatic UC on February 2, 2017, and is also recommended in the NCCN Guidelines. CheckMate 032 was a multicenter, openlabel, 2-stage, multi-arm phase I/II study in patients with UC of the bladder or upper tract. This trial consisted of 2 treatment arms: nivolumab as monotherapy versus combination nivolumab plus ipilim- umab at 2 doses. The smaller phase II trial enrolled 86 patients in the second-line setting, of whom 78 received treatment with nivolumab monotherapy ( $3 \mathrm{mg} / \mathrm{kg}$ intravenously every 2 weeks). The confirmed investigator-assessed ORR was 24.4\% (19/78 patients) with 5 CRs, and the median OS was 9.7 months with a 9-month follow-up. The most common TRAEs were elevated lipase and amylase levels and fatigue. ${ }^{27}$ The larger phase II trial (CheckMate 275) evaluated activity in 265 patients with a median follow-up of 7 months and reported an ORR in 52 patients (19.6\%) overall and, specifically, in 23 of the 81 patients $(28.4 \%)$ who were PD-L1-positive for expression of $\geq 5 \%$. The most common TRAEs were fatigue and diarrhea, with grade $3 / 4$ TRAEs occurring in 48 of 270 total patients (18\%) treated with nivolumab. ${ }^{28}$

\section{Pembrolizumab}

Pembrolizumab, a fully human monoclonal IgG4א isotype antibody that blocks PD-1, was FDA-approved for use in the second-line setting on May 17, 2017. KEYNOTE-012, a phase Ib basket trial evaluating the safety and activity of pembrolizumab $(10 \mathrm{mg} / \mathrm{kg}$ intravenously every 2 weeks) in patients with $\geq 1 \%$ PD-L1 expression on tumor cells, reported that 27 patients assessed for activity had an ORR of 26\% with 3 CRs; common TRAEs were fatigue and edema. ${ }^{29}$ KEYNOTE-045 was a phase III randomized study of 542 patients with metastatic UC that compared pembrolizumab versus investigator's choice of chemotherapy (taxanes) or vinflunine in the second-line setting. The co-primary end points, OS and PFS, were assessed in all patients and in those with PD-L1 expression $\geq 10 \%$. With a median follow-up of 14.5 months, median OS showed significance at 10.3 months (95\% CI, 8.0-11.8) in the pembrolizumab group compared with 7.4 months $(95 \% \mathrm{CI}$, $6.1-8.3$ ) in the chemotherapy group (HR, 0.73; 95\% CI, 0.59-0.91; $P=.002)$. The median OS for patients with PD-L1 expression $\geq 10 \%$ was also significantly different at 8.0 months $(95 \% \mathrm{CI}, 5.0-12.3)$ in the pembrolizumab group compared with 5.2 months (95\% CI, 4.0-7.4) in the chemotherapy group (HR, 0.57; 95\% CI, 0.37-0.88; $P=.005)$. There was not a significant difference in PFS in all patients and those with PD-L1 expression $\geq 10 \%$. Pembrolizumab had fewer TRAEs $(60.9 \%$ vs $90.2 \%){ }^{30}$ Survival benefit and lower toxicity was maintained at follow-up 
at 18.5 months. ${ }^{31}$ Pembrolizumab in the secondline setting is a category 1 recommendation in the NCCN Guidelines. ${ }^{9}$

Pembrolizumab was also granted accelerated approval in the first line for cisplatin-ineligible patients and is supported by the NCCN Guidelines. Results of KEYNOTE-052, a phase II study of 370 cisplatin-ineligible patients, showed an ORR in $27 \%(\mathrm{CI}$ $95 \%, 22-32$ ) among patients with $\geq 4$ months' of follow-up. PFS and OS rates at 6 months were $31 \%$ and $67 \%$, respectively. Duration of response was not reached and the agent was tolerable, with fatigue among the most common AEs (Tables 1 and 2). ${ }^{32}$

\section{Ipilimumab}

The 3-arm CheckMate-032 trial measured the effect of ipilimumab in combination with PD-1 inhibition. The arms consisted of high-dose ipilimumab (3 mg/ $\mathrm{kg}$ ) every 3 weeks for 4 doses plus nivolumab at 1 $\mathrm{mg} / \mathrm{kg}$ every 2 weeks; low-dose ipilimumab (1 mg/ $\mathrm{kg}$ ) every 3 weeks for 4 doses plus nivolumab at $3 \mathrm{mg} /$ kg every 2 weeks; and nivolumab at $3 \mathrm{mg} / \mathrm{kg}$ every 2 weeks alone (which was reported earlier). Each response was compared with historical controls in the second-line setting. Results showed that the ORR for the 26 patients receiving the high-dose ipilimumab combination was 38.5\% (95\% CI, 20.2-59.4), with a CR rate of $3.8 \%$ and a median follow-up of 7.8 months. In the 104 patients receiving the low-dose ipilimumab combination, the ORR was 26\% (95\% CI, 17.9-35.5) and the CR rate was 2.9\%, with a median follow-up of 16.7 months. Toxicity led to discontinuation for $7.7 \%$ of those in the high-dose ipilimumab group and $13.5 \%$ in the low-dose ipilimumab group. All-grade AEs were in $76.9 \%$ of the high-dose ipilimumab group and $84 \%$ of the low-dose ipilimumab group, with the common being pruritus, rash, diarrhea, and thyroid dysfunction; there was 1 treatment-related death secondary to pneumonitis in the low-dose ipilimumab arm. ${ }^{34}$ In summary, the highdose ipilimumab combination resulted in improved response rates compared with the low-dose ipilimumab combination, nivolumab alone, and historical controls. This immunotherapy combination is being

\begin{tabular}{|c|c|c|c|c|c|c|c|}
\hline & Immunotherapy & Phase & Patients, $\mathrm{n}$ & ORR & os & AEs & Reference \\
\hline \multirow[t]{11}{*}{ PD-L1 } & \multirow{5}{*}{$\begin{array}{l}\text { Atezolizumab } \\
(1,200 \mathrm{mg} \text { q3wk })\end{array}$} & II - IMvigor 210 & \multirow[t]{3}{*}{310} & \multirow[t]{3}{*}{$15 \%$} & \multirow[t]{3}{*}{-} & Fatigue $(30 \%)$ & \multirow[t]{3}{*}{21} \\
\hline & & \multirow[t]{2}{*}{ Cohort 2} & & & & Nausea $(14 \%)$ & \\
\hline & & & & & & Decreased appetite (12\%) & \\
\hline & & \multirow{2}{*}{$\begin{array}{r}\text { III - IMvigor } 211 \\
\text { (randomized) }\end{array}$} & \multirow[t]{2}{*}{911} & $23 \%$ & $11.1 \mathrm{mo}^{\mathrm{a}}$ & & \multirow[t]{2}{*}{23} \\
\hline & & & & (8 CRs) & (10.6 mo) & & \\
\hline & \multirow{4}{*}{$\begin{array}{l}\text { Durvalumab } \\
(10 \mathrm{mg} / \mathrm{kg} \text { q2wk) }\end{array}$} & \multirow[t]{2}{*}{$1 / I I$} & \multirow[t]{2}{*}{61} & \multirow[t]{2}{*}{$31 \%$} & \multirow[t]{2}{*}{-} & Fatigue $(39 \%)$ & \multirow[t]{2}{*}{24} \\
\hline & & & & & & MSK pain $(24 \%)$ & \\
\hline & & \multirow[t]{2}{*}{ II } & \multirow[t]{2}{*}{191} & $17.8 \%$ & \multirow[t]{2}{*}{$18.2 \mathrm{mo}$} & Constipation & \multirow[t]{2}{*}{25} \\
\hline & & & & (7 CRs) & & & \\
\hline & \multirow{2}{*}{$\begin{array}{l}\text { Avelumab } \\
\text { (10 mg/kg q2wk) }\end{array}$} & \multirow[t]{2}{*}{ I } & \multirow[t]{2}{*}{44} & $18.2 \%$ & \multirow[t]{2}{*}{$13.7 \mathrm{mo}$} & Fatigue $(31.8 \%)$ & \multirow[t]{2}{*}{26} \\
\hline & & & & (5 CRs) & & $\begin{array}{l}\text { Infusion-related } \\
\text { nausea }\end{array}$ & \\
\hline \multirow[t]{9}{*}{ PD-1 } & \multirow{4}{*}{$\begin{array}{l}\text { Nivolumab } \\
(3 \mathrm{mg} / \mathrm{kg} \text { q2wk) }\end{array}$} & \multirow[t]{3}{*}{ I/II - CheckMate 032} & \multirow[t]{3}{*}{78} & $19 \%$ & \multirow[t]{3}{*}{$9.7 \mathrm{mo}$} & Elevated lipase & \multirow[t]{3}{*}{27} \\
\hline & & & & (5 CRs) & & Elevated amylase & \\
\hline & & & & & & Fatigue & \\
\hline & & II - CheckMate 275 & 270 & $19.6 \%$ & $8.7 \mathrm{mo}$ & & 28 \\
\hline & \multirow{5}{*}{$\begin{array}{l}\text { Pembrolizumab } \\
\text { (200 mg q3wk) }\end{array}$} & \multirow[t]{3}{*}{ I - KEYNOTE-012 } & \multirow[t]{3}{*}{27} & $26 \%$ & - & Pruritus (19.5\%) & 29 \\
\hline & & & & & & Fatigue $(13.9 \%)$ & \\
\hline & & & & & & Nausea $(10.9 \%)$ & \\
\hline & & III - KEYNOTE-045 & 545 & $21.1 \%$ & $10.3 \mathrm{mo}^{\mathrm{c}}$ & & 30,31 \\
\hline & & (randomized) & & & (7.4 mo) & & \\
\hline
\end{tabular}

Abbreviations: AE, adverse events; CR, complete response; HR, hazard ratio; MSK, musculoskeletal; ORR, overall response rate; OS, overall survival. aMeasured in high PD-L1 expression and nonsignificant.

${ }^{b}$ Approved for 240 mg q2wk in February 2017.

'Significantly different (HR, 0.73; 95\% Cl, 0.59-0.91; $P=.002)$. 
Chism

\begin{tabular}{|c|c|c|c|c|c|c|c|}
\hline & Immunotherapy & Phase & Patients, n & ORR & os & AEs & Reference \\
\hline PD-L1 & $\begin{array}{l}\text { Atezolizumab } \\
(1,200 \mathrm{mg} \text { q3wk })\end{array}$ & $\begin{array}{l}\text { II - IMvigor } 210 \\
\text { Cohort } 1\end{array}$ & 119 & $\begin{array}{l}23 \% \\
(9 \% \mathrm{CR})\end{array}$ & - & $\begin{array}{l}\text { Fatigue } \\
\text { Pruritus } \\
\text { Diarrhea }\end{array}$ & 22 \\
\hline PD-1 & $\begin{array}{l}\text { Pembrolizumab } \\
\text { (200 mg q3wk) }\end{array}$ & II - KEYNOTE-052 & 374 & $\begin{array}{l}24 \% \\
(6 \% \mathrm{CR})\end{array}$ & - & Fatigue & 32 \\
\hline
\end{tabular}

Abbreviations: AEs, adverse events; $C R$, complete response; ORR, overall response rate; OS, overall survival

investigated in clinical trials and is not currently approved for bladder cancer treatment.

\section{Epacadostat}

Epacadostat is a novel IDO-1 inhibitor that suppresses systemic tryptophan catabolism. In a phase II part of the ECHO-202/KEYNOTE-037 trial, 40 patients with 0 or $>1$ prior line of treatment received $100 \mathrm{mg}$ of epacadostat twice daily plus $200 \mathrm{mg}$ of pembrolizumab every 3 weeks. Median duration of response with the combination was 30.6 weeks (range, 9.7-93.1); in patients who received 0 or 1 prior therapies ( $75 \%$ of patients), the ORR was $38 \%$ (in all evaluable patients, 35\%). Eleven patients had PD-L1 expression $>1 \%$ (tumor and immune cells). At a median follow-up of 33.8 weeks, there were 14 responses with 3 CRs. Most responses were durable and occurred at the first evaluation point; $70 \%$ of patients experienced at least 1 all-grade $\mathrm{AE}$, with the most common being fatigue and rash; 3 patients had grade 3/4 AEs (severe skin reaction); and there were no treatment deaths. ${ }^{35}$

\section{The PD-L1 Challenge}

PD-L1 expression alone as a predictive biomarker has been controversial. First, there have been 5 checkpoint inhibitor approvals with 4 distinct diagnostic tools to measure PD-L1 expression. PD-L1 expression was based on immunohistochemistry (IHC), with the SP142 assay measuring immune cells with a high expression cutoff of $\geq 5 \%$ in the phase II study examining atezolizumab in post-platinum patients. ${ }^{21}$ Alternately, anti-PD-L1 antibody via IHC staining with a cutoff of $\geq 1 \%$ in tumor cells (for trial entry) and both tumor and immune cells (for biomarker and response) was used in the phase $\mathrm{Ib}$ basket trial evaluating the safety and activity of pembrolizum$\mathrm{ab}$ in the second-line setting. Of note, subsequent pembrolizumab trials in bladder cancer used different methods of interpretation and cutoffs for the PD-L1 assay. The durvalumab trial measured PD-L1 expression using IHC, with the VENTANA SP263 assay (Roche, Tuscon, AZ) measuring both tumor and immune cells.24 The role of PD-L1 is uncertain due to variation in assays used and the criteria for positive expression with varying sensitivities. PDL1 expression may be heterogeneous within tumors and between the primary tumor and metastases. ${ }^{36}$ Potential candidate biomarkers may include mutational burden and genomic subtypes. For example, Rosenberg et $\mathrm{al}^{21}$ also measured mutation burden in patients enrolled in the atezolizumab post-platinum study and found that mutation burden was significantly increased in responders versus nonresponders in cohorts 2 and $1 .{ }^{22}$ Cohort 2 patients were classified based on The Cancer Genome Atlas (TCGA) into 4 subtypes with PD-L1 expression highly enriched for the basal subtype. All TCGA subtypes had responses to atezolizumab, but the luminal cluster II had a significantly higher response. ${ }^{21}$

\section{Ongoing Clinical Trials and Conclusions}

The rise of immunotherapy need not equate to the sudden demise of the chemotherapy backbone in metastatic UC. Challenges persist and questions remain to be explored. Which patient with metastatic UC will experience response to cisplatin-based chemotherapy and/or immunotherapy? IMvigor 130, a 3-arm trial investigating atezolizumab plus chemotherapy versus chemotherapy alone versus atezolizumab in the first-line setting, addresses this question (ClinicalTrials.gov identifier: NCT02807636). Exploratory correlatives including bladder cancer subtypes and PD-1 expression will be required. Ongoing prospective studies are investigating tumor characteristics in patients treated with nivolumab alone or 
in combination, with a goal of identifying the relationship between tumor mutation burden and predicted neoantigen burden (NCT02553642). Will the sequence of chemotherapy with immunotherapy matter? JAVELIN Bladder 100, a study of avelumab in metastatic UC comparing maintenance treatment in patients without progression after completion of first-line chemotherapy with avelumab plus BSC versus BSC alone is ongoing (NCT02603432). A similarly designed trial using pembrolizumab is ongoing (NCT02500121). Will combination immunotherapies be more effective than PD-1 or PDL1 inhibition alone? Both CheckMate-901, the nivolumab plus ipilimumab study in the first-line setting (NCT03036098), and DANUBE, studying durvalumab combined with tremelimumab versus stan- dard of care or durvalumab alone (NCT02516241), are investigating this question.

The continued success of the UC immunotherapy treatment model for metastatic bladder cancer will be contingent in part on the following: (1) an immunotherapy that supplements rather than supplants the chemotherapy cornerstone with careful use of predictive biomarkers; (2) an application of immunotherapy clinical research "lessons" from other tumor types such as melanoma and lung cancer; (3) incorporation and development of translational research through novel immunotherapy mouse models; and (4) the maintenance of financial sustainability by the healthcare system and avoidance of financial toxicity by the patients themselves.

\section{References}

1. Siegel RL, Miller KD, Jemal A. Cancer statistics, 2017. CA Cancer J Clin 2017;67:7-30.

2. Cancer of the Urinary Bladder-SEER Stat Fact Sheets. Available at: http://www.cancer.org/cancer/bladercancer/detailedguide/bladder-cancerkey-statistics. Accessed June 1, 2017.

3. Galsky MD, Hahn NM, Rosenberg J, et al. A consensus definition of patients with metastatic urothelial carcinoma who are unfit for cisplatinbased chemotherapy. Lancet Oncol 2011;12:211-214.

4. Loehrer PJ Sr, Einhorn LH, Elson PJ, et al. A randomized comparison of cisplatin alone or in combination with methotrexate, vinblastine, and doxorubicin in patients with metastatic urothelial carcinoma: a cooperative group study. J Clin Oncol 1992;10:1066-1073.

5. Sternberg CN, de Mulder PH, Schornagel JH, et al. Randomized phase III trial of high-dose-intensity methotrexate, vinblastine, doxorubicin, and cisplatin (MVAC) chemotherapy and recombinant human granulocyte colony-stimulating factor versus classic MVAC in advanced urothelial tract tumors: European Organization for Research and Treatment of Cancer protocol no. 30924. J Clin Oncol 2001;19:2638-2646.

6. Sternberg CN, de Mulder P, Schornagel JH, et al. Seven year update of an EORTC phase III trial of high-dose intensity M-VAC chemotherapy and G-CSF versus classic M-VAC in advanced urothelial tract tumours. Eur J Cancer 2006;42:50-54.

7. von der Maase H, Sengelov L, Roberts JT, et al. Long-term survival results of a randomized trial comparing gemcitabine plus cisplatin, with methotrexate, vinblastine, doxorubicin, plus cisplatin in patients with bladder cancer. J Clin Oncol 2005;23:4602-4608.

8. Bellmunt J, von der Maase H, Mead GM, et al. Randomized phase III study comparing paclitaxel/cisplatin/gemcitabine and gemcitabine/cisplatin in patients with locally advanced or metastatic urothelial cancer without prior systemic therapy: EORTC Intergroup Study 30987. J Clin Oncol 2012;30:1107-1013

9. Spiess PE, Agarwal N, Bangs R, et al. NCCN Clinical Practice Guidelines in Oncology: Bladder Cancer, Version 5.2017. Accessed September 2, 2017. To view the most recent version of the guidelines, visit NCCN.org.

10. De Santis M, Bellmunt J, Mead G, et al. Randomized phase II/IIl trial assessing gemcitabine/carboplatin and methotrexate/carboplatin/ vinblastine in patients with advanced urothelial cancer who are unfit for cisplatin-based chemotherapy: EORTC study 30986. J Clin Oncol 2012;30:191-199.

11. Bellmunt J, Theodore C, Demkov $T$, et al. Phase III trial of vinflunine plus best supportive care compared with best supportive care alone after a platinum-containing regimen in patients with advanced transitional cell carcinoma of the urothelial tract. J Clin Oncol 2009;27:4454-4461.

12. Dreicer R, Gustin DM, See WA, Williams RD. Paclitaxel in advanced urothelial carcinoma: its role in patients with renal insufficiency and as salvage therapy. J Urol 1996;156:1606-1608.
13. Sweeney CJ, Roth BJ, Kabbinavar FF, et al. Phase II study of pemetrexed for second-line treatment of transitional cell cancer of the urothelium. J Clin Oncol 2006;24:3451-3457.

14. Lorusso V, Pollera CF, Antimi M, et al. A phase II study of gemcitabine in patients with transitional cell carcinoma of the urinary tract previously treated with platinum. Italian Co-operative Group on Bladder Cancer. Eur J Cancer 1998;34:1208-1212.

15. Hodi FS, O'Day SJ, McDermott DF, et al. Improved survival with ipilimumab in patients with metastatic melanoma. $\mathrm{N}$ Engl J Med 2010;363:711-723.

16. Larkin J, Chiarion-Sileni V, Gonzalez R, et al. Combined nivolumab and ipilimumab or monotherapy in untreated melanoma. N Engl J Med 2015;373:23-34

17. Robert C, Schachter J, Long GV, et al. Pembrolizumab versus ipilimumab in advanced melanoma. N Engl J Med 2015;372:2521-2532.

18. Alexandrov LB, Nik-Zainal $S$, Wege DC, et al. Signatures of mutational processes in human cancer. Nature 2013;500:415-421.

19. Tumeh PC, Harview CL, Yearley JH, et al. PD-1 blockade induces responses by inhibiting adaptive immune resistance. Nature 2014;515:568-571.

20. Moon YW, Hajjar J, Hwu P, Naing A. Targeting the indoleamine 2,3-dioxygenase pathway in cancer. J Immunother Cancer 2015;3:51.

21. Rosenberg JE, Hoffman-Censits J, Powles T, et al. Atezolizumab in patients with locally advanced and metastatic urothelial carcinoma who have progressed following treatment with platinum-based chemotherapy: a single-arm, multicentre, phase 2 trial. Lancet 2016;387:1909-1920.

22. Balar AV, Galsky MD, Rosenberg JE, et al. Atezolizumab as first-line treatment in cisplatin-ineligible patients with locally advanced and metastatic urothelial carcinoma: a single-arm, multicentre, phase 2 trial. Lancet 2017;389:67-76.

23. Powles T. IMvigor211: a phase III randomized study examining atezolizumab vs. chemotherapy for platinum-treated advanced urothelial carcinoma. Presented at the 2nd EACR AACR SIC Special Conference 2017; June 27, 2017; Florence, Italy.

24. Massard C, Gordon MS, Sharma S, et al. Safety and efficacy of durvalumab (MEDI4736), an anti-programmed cell death ligand-1 immune checkpoint inhibitor, in patients with advanced urothelial bladder cancer. J Clin Oncol 2016;34:3119-3125.

25. Hahn NM, Powles T, Massard C, et al. Updated efficacy and tolerability of durvalumab in locally advanced or metastatic urothelial carcinoma (UC) [abstract]. J Clin Oncol 2017;35(Suppl):Abstract 4525.

26. Apolo AB, Infante JR, Balmanoukian A, et al. Avelumab, an antiprogrammed death-ligand 1 antibody, in patients with refractory metastatic urothelial carcinoma: results from a multicenter, phase Ib study. J Clin Oncol 2017;35:2117-2124

27. Sharma $\mathrm{P}$, Callahan $\mathrm{MK}$, Bono $\mathrm{P}$, et al. Nivolumab monotherapy in recurrent metastatic urothelial carcinoma (CheckMate 032): a 
Chism

multicentre, open-label, two-stage, multi-arm, phase 1/2 trial. Lancet Oncol 2016;17:1590-1598.

28. Sharma P, Retz M, Siefker-Radtke A, et al. Nivolumab in metastatic urothelial carcinoma after platinum therapy (CheckMate 275): a multicentre, single-arm, phase 2 trial. Lancet Oncol 2017;18:312-322.

29. Plimack ER, Bellmunt J, Gupta S, et al. Safety and activity of pembrolizumab in patients with locally advanced or metastatic urothelial cancer (KEYNOTE-012): a non-randomised, open-label, phase $1 \mathrm{~b}$ study. Lancet Oncol 2017;18:212-220.

30. Bellmunt J, De Wit E, Vaughn DJ, et al. Pembrolizumab as second-line therapy for advanced urothelial carcinoma. N Engl J Med 2017;376:10151026.

31. Bajorin DF, De Wit R, Vaughn DJ, et al. Planned survival analysis from KEYNOTE-045: Phase 3, open-label study of pembrolizumab (pembro) versus paclitaxel, docetaxel, or vinflunine in recurrent, advanced urothelial cancer (UC) [abstract]. J Clin Oncol 2017;35(Suppl):Abstract 4501.

32. Balar AV, Catellano DE, O'Donnell PH, et al. Pembrolizumab as firstline therapy in cisplatin-ineligible advanced urothelial cancer: results from the total KEYNOTE-052 study population [abstract]. J Clin Oncol 2017;35(Suppl):Abstract 284.

33. Galsky MD, Hahn NM, Albany C, et al. Phase II trial of gemcitabine + cisplatin + ipilimumab in patients with metastatic urothelial cancer [abstract]. J Clin Oncol 2016;34(Suppl):Abstract 357.

34. Sharma P, Callahan MK, Calvo E, et al. Efficacy and safety of nivolumab plus ipilimumab in metastatic urothelial carcinoma: first results from the phase I/II CheckMate 032 study. Presented at the 216 SITCF Annual Meeting; November 9-13, 2016; National Harbor, Maryland.

35. Smith DC, Gajewski T, Hami O, et al. Epacadostat plus pembrolizumab in patients with advanced urothelial carcinoma: preliminary phase I/II results of ECHO-202/KEYNoTE-037 [abstract]. J Clin Oncol 2017;35(Suppl):Abstract 4503.

36. Madore J, Vilain RE, Menzies AM, et al. PD-L1 expression in melanoma shows marked heterogeneity within and between patients: implications for anti-PD-1/PD-L1 clinical trials. Pigment Cell Melanoma Res 2015;28:245-253.

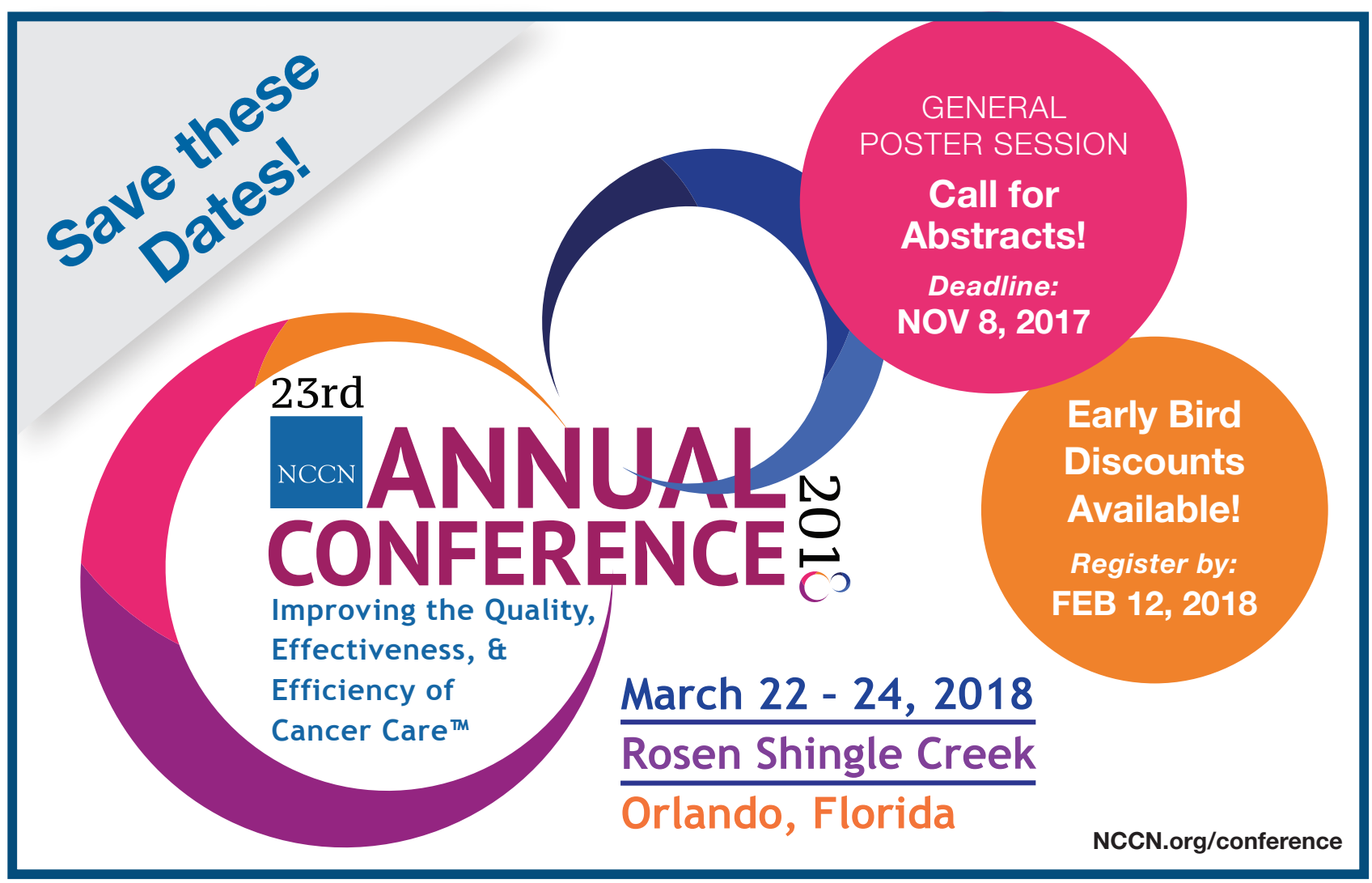

